Note

\title{
Maximal Stationary Metabolic Rate in Free-Moving Rats
}

\author{
Naoko Komenami ${ }^{1}$ and Masamitsu Miyoshi ${ }^{2, *}$ \\ ${ }^{1}$ Graduate School of Human Culture and ${ }^{2}$ Department of Food Science and \\ Nutrition, Nara Women's University, Nara 630, Japan
}

(Received May 23, 1996)

\begin{abstract}
Summary This paper provides a procedure for estimating the maximal stationary metabolic rate (SMRmax) in free-moving rats. The SMRmax was estimated by simultaneously comparing energy expenditure with motor activity measured at $10 \mathrm{~min}$ intervals for $24 \mathrm{~h}$. The SMRmax was close to but naturally higher than the resting metabolic rates determined by other conventional methods. The coefficient of variation for 3 consecutive days was below 3\%. The ratio of SMRmax to daily minimum energy expenditure was 1.25 in young rats and 1.19 in adult rats. SMRmax is a useful key parameter for analyzing daily energy expenditure and behavior.
\end{abstract}

Key Words maximal stationary metabolic rate, energy expenditure, rats

Energy metabolism has been a target of research in nutrition for controlling energy balance to prevent and treat obesity, and maintaining health or strengthening physical fitness (1-3). Our purpose of studying energy metabolism, however, is to facilitate a more detailed behavior analysis from the viewpoint of a $24 \mathrm{~h}$ energy expenditure pattern in small animals as a human model (4). We previously developed an indirect calorimeter laid on a mass analyzer and a computer which made it possible to measure the daily energy expenditure of small animals in respiration chambers (5). In addition, we have proposed a new procedure for estimating daily minimum energy expenditure (MEE) by a regression procedure (6). In this paper, we present a new concept of maximal stationary metabolic rate (SMRmax) in rats and a procedure for its estimation under free-living conditions. SMRmax is defined as the largest energy expenditure per unit of time in a stationary state. It does not meet the standard conditions of resting metabolic rate (RMR), but is a key parameter for classifying daily energy expenditure into such components as resting and moving.

\section{Methods}

Experimental animals. Male Wistar rats were kept in a room at $25^{\circ} \mathrm{C}$ with a $14 \mathrm{~h}$ light period $(5: 00-19: 00)$ and $10 \mathrm{~h}$ dark period $(19: 00-5: 00)$, and allowed

\footnotetext{
* To whom correspondence should be addressed.
} 
free access to water and food (Chow diet: MF type, Oriental Bioservice). Their ages ranged from 7 to 35 weeks depending upon experiments, but data were compared at same ages.

Measurement of energy metabolism. Two rats were individually placed in an open-circuit plastic respiration-chamber $(24 \times 46 \times 18 \mathrm{~cm})$ connected to a gas mass spectrometer (WSMR-1400, Westron). The air flow was controlled to $4 \mathrm{~L} / \mathrm{min}$. A gas analysis was carried out from 10:00 to 9:00 on the following day. Energy expenditure was measured at $10 \mathrm{~min}$ intervals (5) and MEE was calculated as described in a previous paper (6).

Measurement of activity. Locomotor activity (counts/10 min) was measured by an Animex (III type, Shimadzu) laid under each respiration chamber. Activity was also monitored by a videotape camera and evaluated as moving distance $(\mathrm{m} / 10$ $\min$ ) by image analysis.

Estimation of SMRmax. SMRmax is defined in this paper as the largest daily stationary energy expenditure per $10 \mathrm{~min}$ in free-moving rats. The unit of time, however, can be changed depending upon experimental purposes and conditions. The SMRmax was estimated by a regression procedure as shown in a previous paper (6) and charted on a graph of sorted daily energy expenditure together with activity data per $10 \mathrm{~min}$ as shown in Fig. 1. Smaller values on the lefthand side of the graph can be regarded as representing data at the stationary level. The resting data give a smooth curve which goes up gradually from left to right. There is a point on the graph where energy expenditure goes up more sharply. This point coincides well with the point of significant increase in activity measured by either locomotion or image analysis. So, energy expenditure at this point, which can be determined by differential procedure, is taken as SMRmax. The problem of what a rat is doing at SMRmax was largely solved in this study by visual observation of the rat's behavior.

Estimation of RMRs. RMRs of 3 types were measured by conventional methods as briefly described in Table 1 .

Results

Data of a 10-week-old rat in Fig. 1 presents evidence for the rationale of SMRmax described in Methods. A differential calculation can detect even a small change of energy expenditure, which separates stationary level from active level, on a sorted graph of daily energy expenditure. Small activities in this stationary range are negligible since they were detected only at the highest sensitivity of the Animex. Activity counts at the stationary level can be made smaller if a smaller unit of time and/or a lower sensitivity is employed. However, since the goal of our study was to analyze daily behavior quantitatively from the viewpoint of energy expenditure, it seemed appropriate to measure energy expenditure and activity at a $10 \mathrm{~min}$ interval.

Figure 2 shows the results of visual observation of rat behavior during a light period. The visual description is supplemented by two types of quantitative data; energy expenditure and locomotor activity. SMRmax measured beforehand is also 


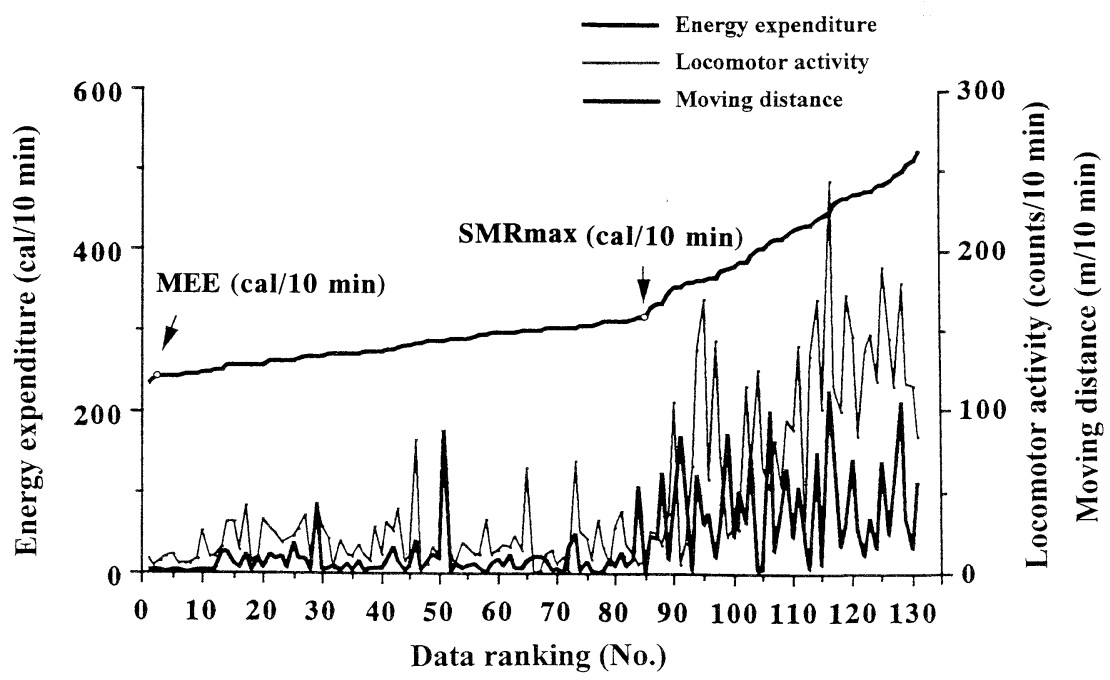

Fig. 1. An example of daily energy expenditure sorted in an ascending order with corresponding locomotor activity and moving distance of a rat (10 weeks of age). MEE and SMRmax were estimated by a regression procedure (see text) and plotted on this graph of sorted energy expenditure.

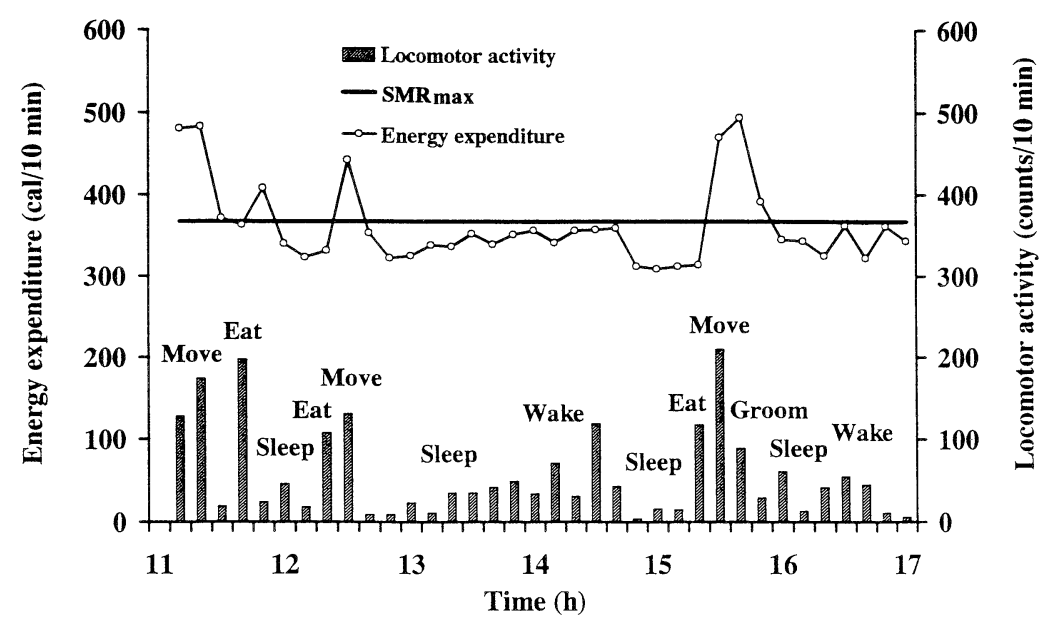

Fig. 2. Examples of SMRmax, diurnal patterns of energy expenditure, locomotor activity and performance of a rat. The performance of a rat (35 weeks of age), in a respiration chamber monitored with an observation camera and recorded on videotape between 11:00 and 17:00 in a light period, was classified into Move, Eat, Groom, Sleep (closed eyes) and Wake (opened eyes) to examine what the rat was doing at the SMRmax level. 
shown in the figure. The SMRmax comes around a metabolic rate at a relatively high level of energy expenditure in either sleeping or motionless states, which is in accord with its definition.

Table 1 compares SMRmax with various RMRs of the same 6 rats, young and adult, estimated on three successive days by three different methods. SMRmax and RMR1 of the young rats were approximately 1.25 times as large as MEE. RMR2 and 3 were a little smaller being about 1.18 times as large as MEE. The coefficient of variation (CV) of RMR2 was the smallest (1.4\%) and that of RMR3 the largest $(5.4 \%)$ in the young rats. The CVs of SMRmax and RMR1 were around $2 \%$. SMRmax and all the RMRs of the adult rats came to a range between 1.1 and 1.2 times as large as MEE. Those of the young rats had a wider range in this respect, which could occur because the metabolic rate is higher in young rats than in adult ones even at rest. The CVs were stable in all the RMRs, with particularly smaller values in RMR1 (0.9\%) and RMR2 (1.0\%) in the adult rats. No statistical difference was found among SMRmax and the three RMRs in any group on any day. SMRmax estimated by our new method, however, was naturally a little larger than the RMRs estimated by the conventional methods and the relative value of

Table 1. Daily variation of MEE, SMRmax and RMRs for successive days in young and adult rats.

\begin{tabular}{lllc}
\hline & $\begin{array}{c}\text { Mean } \pm \text { SD } \\
(\text { cal/10 min })\end{array}$ & $\begin{array}{c}\text { CV } \\
(\%)\end{array}$ & Ratio to MEE \\
\hline Young rats (7 weeks) & & & \\
MEE & $236 \pm 2$ & 0.8 & 1.00 \\
SMRmax & $295 \pm 5$ & 1.7 & 1.25 \\
RMR1 & $291 \pm 7$ & 2.4 & 1.24 \\
RMR2 & $277 \pm 4$ & 1.4 & 1.17 \\
RMR3 & $279 \pm 15$ & 5.4 & 1.18 \\
Adult rats (34 weeks) & & & \\
MEE & $272 \pm 3$ & 1.1 & 1.00 \\
SMRmax & $323 \pm 9$ & 2.8 & 1.19 \\
RMR1 & $316 \pm 3$ & 0.9 & 1.16 \\
RMR2 & $309 \pm 3$ & 1.0 & 1.14 \\
RMR3 & $307 \pm 8$ & 2.6 & 1.13 \\
\hline
\end{tabular}

Values are mean \pm SD for 6 rats. RMR1, resting metabolic rate estimated from the smallest energy expenditure per $10 \mathrm{~min}$ during a dark period by the same regression procedure as that employed for the calculation of MEE. RMR2, resting metabolic rate estimated from the energy expenditure per $10 \mathrm{~min}$ at a zero count level of locomotor activity by a regression curve drawn between count data of locomotor activity per $10 \mathrm{~min}$ and energy metabolic rate per $10 \mathrm{~min}$. RMR3, resting metabolic rate estimated from mean energy expenditure per $10 \mathrm{~min}$ during $1 \mathrm{~h}$ in the middle of a light period, between $11: 00$ and 12:00, when rats were at rest. CV, coefficient of variation in values of 3 days. There was no significant difference between SMRmax and RMRs according to one-way ANOVA. 
SMRmax to MEE seems reasonable.

Figure 3 shows total resting times per day of young and adult rats estimated by summing up the time corresponding to all the $10 \mathrm{~min}$ data blocks smaller than SMRmax. The resting times were around 8 to $10 \mathrm{~h}$ per day, a little shorter than the generally admitted sleeping time of rats.

\section{Discussion}

Ceesay et al. followed metabolic changes in humans during $24 \mathrm{~h}$ and visually described their behavior (7). In this study, we carried out a similar experiment using rats. In the analysis of a $24 \mathrm{~h}$ energy expenditure pattern, it is important and useful to detect an energy level of SMRmax. In this paper, a new method was presented for estimating SMRmax in rats. The reliability of SMRmax was justified by a comparison of energy expenditure with locomotor activity and visual description of rat action at SMRmax. A comparison of SMRmax with RMRs estimated by conventional methods indicated that SMRmax gave a reasonable highest value at rest. Small CVs reflect stability and reliability of the present SMRmax measurement. The actual value of the present SMRmax was close to the results by Gleeson et al. on rats of similar ages $(8)$. The average resting time of the rats estimated from the viewpoint of SMRmax in this study was between 8 and $10 \mathrm{~h}$ per day, which is rather shorter than the $12-13 \mathrm{~h}$ reported by Jouvet et al. (9) and Meddis (10). Exchange of the rats, food and water during the light period just before the start of measurement might have caused the shorter resting time.

Various ideas of component analysis of daily energy expenditure have been presented for both humans and animals $(11-13)$. We have already reported a method for MEE estimation in rats (6). The present SMRmax estimation leads to

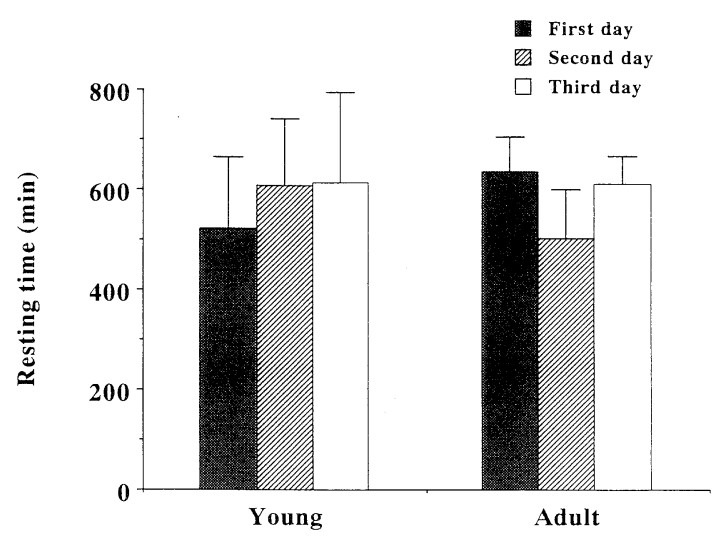

Fig. 3. Resting time estimated from SMRmax. Values are mean \pm SD for 6 rats. Resting time was calculated from the number of data blocks of 10 min energy expenditures below SMRmax in young (7 weeks) and adult (34 weeks) rats. There was no significant difference either among the 3 daily values in each group or in the resting times between young and adult rats (ANOVA). 
the measurement of daily activity which is the sum of energy expenditure above SMRmax. Therefore, a simple but quantitative analysis of daily energy expenditure and behavior in small animals is now possible by taking MEE and SMRmax into consideration.

We thank Ms. C. Nakajima and Ms. A. Kato for their skilled technical assistance.

\section{REFERENCES}

1) Foster GD, Wadden TA, Mullen JL, Stunkard AJ, Wang J, Feurer ID, Pierson RN, Yang MU, Presta E, Iitallie TBV, Lemberg PS, Gold J. 1988. Resting energy expenditure, body composition, and excess weight in the obese. Metabolism 37: 467472.

2) Mansell PI, Macdonald IA. 1988. The effect of underfeeding on the physiological response to food ingestion in normal weight women. Br J Nutr 60: 39-48.

3) Weigle DS, Sando KJ, Lverius PH, Monsen ER, Brunzell JD. 1988. Weight loss leads to a marked decrease in nonresting energy expenditure in ambulatory human subjects. Metabolism 37: 930-936.

4) Miyoshi M, Tsukamoto I, Seo YJ, Toyohara M, Koriyama K, Maeda N, Kotani M. 1989. Analysis of behavior of small animals from a viewpoint of energy consumption and its nutritional applications. In: Abstracts of the 14th International Congress of Nutrition, p 347.

5) Komenami N, Miyoshi M. 1992. Energy expenditure in rats measured using an indirect calorimeter equipped with a mass spectrometer. Annu Rep Grad Sch Human Cult 8: 101-110.

6) Komenami N, Yamamoto Y, Miyoshi M. 1995. Estimation of daily minimum energy expenditure in free-moving rats and mice. J Nutr Sci Vitaminol 41: 395-407.

7) Ceesay SM, Prentice AM, Day KC, Murgatroyd PR, Goldberg GR, Scott W, Spurr GB. 1989. The use of heart rate monitoring in the estimation on energy expenditure: a validation study using indirect whole body calorimetry. Br J Nutr 61: 175-186.

8) Gleeson M, Brown JF, Waring JJ. 1982. The effects of physical exercise on metabolic rate and dietary induced thermogenesis. Br J Nutr 47: 173-181.

9) Jouvet MD, Astic L, Lacote D. 1970. Ontogenesis of the states of sleep in rat, cat, and guinea pig during the first postnatal month. Dev Psychobiol 2: 216-239.

10) Meddis R. 1977. The Sleep Instinct. Routledge and K. Paul, London.

11) Jequier E, Schutz Y. 1983. Long-term measurements of energy expenditure in humans using a respiration chamber. Am J Clin Nutr 38: 989-998.

12) Schutz Y, Bessard T, Jequier E. 1984. Diet-induced thermogenesis measured over a whole day in obese and non-obese women. Am J Clin Nutr 40: 542-552.

13) Poehlman ET. 1990. Regulation of energy expenditure in aging humans. Annu Rev Nutr 10: 255-275. 